\title{
The radiation footprint on the pediatric trauma patient
}

\author{
Raquel M. Schears ${ }^{1}$, Zainab Farzal ${ }^{2}$, Zehra Farzal ${ }^{3}$ and Anne C. Fischer ${ }^{4^{*}}$
}

\begin{abstract}
Background: The actual baseline of radiation exposure used in evaluating pediatric trauma is not known and has relied on estimates in the literature that may not reflect clinical reality. Our objectives were to determine the baseline amount of radiation delivered in a pediatric trauma evaluation and correlate radiation exposure with trauma activation status to identify the cohort most at risk.

Methods: We retrospectively evaluated trauma patients $(N=1050)$ at an independent Level I children's hospital for each level of trauma activation (consults, alerts, stats) from June 2010 to January 2011. Those patients with full dosimetry $(N=215)$ were analyzed for demographics, mechanism of injury, Injury Severity Score, imaging modalities, and total effective radiation dosages during the full trauma assessment from the time of injury to discharge.

Results: Demographics included gender (143 males, 72 females) and average age (5.5 years [range $<1-16]$ ). The most radiation was conferred from CTs and greatest in trauma stats, followed by alerts, then consults ( $p<0.001$ for stat and alert doses compared to consults). Repeated imaging was common: 35\% of stats had 2-3 CTs and 40\% had 4-10 CTs (range 0-10 CTs). The average non-accidental trauma consult utilized four times as many CTs as the average consult ( $p=0.002$ ). Most outside hospital CTs (66\%) delivered more radiation: $50.0 \%$ were at least double the standard pediatric dosage.

Conclusions: This study is the first to identify the actual baseline of radiation exposure for one trauma evaluation and correlate radiation exposure with trauma activation status. Factors associated with highest radiation include stat activations, suspected non-accidental traumas (NAT), and outside hospital system imaging.
\end{abstract}

\section{Background Introduction}

The overuse of ionizing radiation in radiographic imaging is a timely and important topic of discussion in the lay press and the medical community. The era of diagnostic imaging changed significantly with the availability of computed tomography (CT) scans. Beginning in the late 1970s, CT scans became highly preferable given their accessibility and ease of scanning. As a result, there was a surge in the use of CT imaging over the past several decades. The number of CT scans obtained annually in the USA increased approximately 40-fold, from 2 million scans in 1980 to 81.2 million scans by 2014, grossly in excess of the growth of the population [1,2]. This trend has resulted in increased radiation exposure among patients

\footnotetext{
* Correspondence: annefischermdphd@gmail.com

${ }^{4}$ Florida Atlantic University/St. Mary's Medical Center, 927 45th Street, Suite 301, West Palm Beach, FL 33407, USA

Full list of author information is available at the end of the article
}

which is concerning since it is thought that up to $30 \%$ of CT scans performed may be unnecessary [3].

\section{Importance}

The link between ionizing radiation and oncogenesis is well known. A hypothetical model based on the cumulative exposure to what was once perceived to be a low and "unconcerning" threshold at the time has now shown that radiation at all levels is cumulative across the population [4]. Children are particularly prone to developing malignancies secondary to ionizing radiation for two reasons. First, they are more radiosensitive due to the presence of rapidly dividing cells in their bodies [5]. Second, children have a longer lifespan during which the malignancy may manifest $[5,6]$. It is postulated most radiation-induced malignancies are dormant for at least 40 years following exposure $[7,8]$. There is growing concern that CT imaging is overused, and it is estimated that up to $2 \%$ of all US cancers may now be attributable 
to the radiation from CT examinations $[1,4]$. This was highlighted for the first time in a retrospective cohort review which showed the incidence of 1 excess case of a brain tumor and 1 excess case of leukemia for every 10,000 head CT scans performed among patients under the age of 10 in the 10 years following a single scan [9]. While this study analyzed tumor rates from an epidemiological perspective and not an individual risk per one CT scan, it highlighted the importance of acknowledging the detectable incidence of cancer and the impact of cumulative radiation exposure per child even if only measurable on a population basis. As a result of increased awareness of the potential hazards involved, there is a rising initiative to diminish radiation exposure particularly in children who are most affected by it.

Injury is currently the leading cause of morbidity and mortality among children [10]. In 2010, the leading cause of death in children aged 1-19 years was injuryrelated according to the CDC [11]. Every year, more than 10 million children are evaluated and treated in emergency departments for traumatic injuries around the USA [12]. As in other patient populations, the use of CT scans has increased in pediatric trauma patients [13]. The high index of suspicion in trauma has created a paradigm of reflexively scanning patients, even those who are asymptomatic, to ensure identifying all potential injuries presumed by the mechanism of injury.

\section{Goals of this investigation}

Our goal was to identify the actual radiation doses used in the evaluation of a pediatric trauma patient from the time of injury since the numbers available in the literature are hypothetical extrapolated doses. Our hypothesis was that trauma activation status is a consistent determination of the acuity of trauma across institutions that best correlates with the amount and type of imaging used to identify potential injuries often presumed by mechanism. We correlated the amount of imaging with trauma activation status in order to identify the amount of radiation per a given activation status to identify the subset of patients most at risk for higher exposures and the subset most amenable to the consideration of reduced imaging strategies. We chose a time period (1) that pre-dated the induction of many reiterative and variable changes to reduce radiation such as newer $\mathrm{CT}$ modalities, as flash $\mathrm{CT}$ scans, and implementation of pediatric-specific lower dose scanning protocols and (2) when documentation of the dosimetry was first available for collection to obtain the most realistic level of exposure, given the serious epidemiologic implications for the future.

Most studies [14-16] have focused on a mean effective dose for the overall population, averaging all levels of trauma acuity. Institutions vary from each other in respect to their trauma acuity, and therefore, this overall averaging on estimations in the literature underestimates the radiation dose for stat patients and overestimates exposure in non-urgent trauma consult patients.

\section{Methods}

\section{Study design and setting}

Our study was an Institutional Board Review (IRB)-approved retrospective review of the trauma registry at Children's Medical Center in Dallas, an independent Level I children's hospital. We evaluated the full encounter from time of injury, including initial outside hospital evaluation for transferred patients, in all trauma activations $(N=1050)$ which included stats, alerts, and consults from June 1, 2010, to January 31, 2011. This time interval represented the earliest time period during which complete dosimetry was initially captured at our hospital prior to the multitude of iterative and variable changes introduced through quality improvement in imaging to reduce radiation exposure.

\section{Selection of patients and outcomes}

The trauma population at Dallas Children's Medical Center was stratified into the standard three activation statuses based on specific criteria (Table 1). Briefly, trauma consults reflect the lowest activation level, followed by trauma alerts which are triaged at a greater level of concern, while trauma stats represent the highest activation level with potentially life-threatening injuries. This classification is based on the American College of Surgeons Committee on Trauma recommendations [12]. Of the 1050 patients in the 7-month time frame, 739 were admitted to the inpatient service. A total of 60 trauma stat patients with available dosimetry were seen in this time period. Due to a disproportionately higher volume of trauma consult and alert patients, only those presenting in the first 2 months were included for comparison (alerts $N=85$, consults $N=70$ ) to approximate the numbers in the trauma stat subset. Two hundred fifteen patients met inclusion criteria (after the exclusion of 32 patients with missing outside hospital (OSH) imaging) with complete dosimetry available from the time of injury to trauma evaluation on transfer and hospitalization. These patients' records were analyzed for demographics, mechanism of injury, Injury Severity Score (ISS), imaging modalities, total radiation exposure including the number of $\mathrm{CT}$ scans and plain films, and $\mathrm{CT}$ radiation dosages in milliSieverts (mSvs). All on-site imaging including the number of CT scans, plain films, and CT doses were taken directly from the Children's Medical Center radiology records.

Being a Level I trauma service, our trauma admissions included patients transferred to our institution's 
Table 1 Children's Medical Center Dallas trauma activation criteria

Trauma Stat

Traumatic cardiopulmonary arrest from penetrating trauma

Traumatic injury with signs of shock

Penetrating injuries to the head, neck, chest, abdomen or pelvis (excludes lacerations in the stable patient)

Respiratory distress secondary to trauma, respiratory compromise/ obstruction and/or intubation on scene

Neurological injury with a GCS equal to or less than 8 without sedation

Suspected spinal cord injury: associated with flaccidity, are flexia or unexplained hypotension

Crush or Amputation proximal to the wrist or ankle with signs of shock

Any trauma transfer with respiratory and/or hemodynamic instability and/or GCS equal to or less than 8 without sedation or paralytics and/ or patients receiving blood to maintain vital signs

Any intubated trauma transfer

Emergency physician's discretion

Trauma Alert

Traumatic cardiopulmonary arrest from blunt trauma

Motor Vehicle Crashes (includes ATV's) with reported history of: ejection of the patient from the vehicle, prolonged extrication (> 20 minutes), a rollover collision, death of an occupant in same vehicle

Neurological injuries with a GCS of 9 to 14

Hanging or strangulation mechanisms

Auto-Pedestrian or Auto-Bike Crashes involving speeds equal to or greater than $20 \mathrm{mph}$

Falls greater than 2nd story or 20 feet

Bilateral femur fractures or 3 or more long bone fractures

Crush injuries to chest or abdomen

Crush or Amputation injuries proximal to the wrist or ankle in the stable patient with fracture or significant tissue loss

Significant lacerations to head or neck in the stable patient

- Lacerations that are deep or with significant tissue loss

Any transfer with a grade IV solid organ injury or two or more solid organ injuries

Trauma Consult

Child abuse cases to be admitted

Any trauma related injury where two or more systems are involved

Any patient that has a single system injury that requires admission and the mechanism is an MVC, MPC, ATV

emergency department after a prior evaluation at an outside hospital. Patients transferred from an outside hospital who were included in the study had imaging and radiation data previously uploaded into our hospital's records for a total of 61 outside CT scans. Transferred patients with no recorded radiation dose in our electronic medical records were excluded from the study.

\section{Radiation dose measurements}

In order to calculate effective radiation doses in milliSieverts, precise DLP (dose-length product) measurements were taken for each CT scan performed from Children's Medical Center radiology records. A dose-length product is a measure of radiation exposure due to CTs which takes both the amount of radiation used as well as the length of the CT scan into account [17]. The DLP value was multiplied by conversion factors provided in the literature by Shrimpton et al [18] to adjust for patient age and provided the final effective radiation doses in milliSieverts. While several sets of conversion factors exist, this is the most widely used method and was thus utilized in our study [19].

\section{Statistical analysis}

Student's $t$ tests were used to determine the statistical significance of (a) radiation dose from CT scans and (b) number of CT scans performed in the subgroups compared to the trauma consults. The level of significance was set at $p<0.05$. To avoid introducing a confounding variable in the trauma cohorts, non-accidental traumas (NATs) were examined as a distinct category. Of note, no statistical analysis was done for the subcategory of NAT alerts due to an $N$ of 2 .

\section{Results}

\section{Characteristics of study subjects}

The demographics, Injury Severity Scores (ISSs), and mechanisms of injury comparing consults, alerts, and stats are presented in Table 2. Most trauma consults were due to fractures from falls. Among alerts, the majority of patients presented due to car accidents or falls while the stats were mostly either car accident injuries or NATs.

Table 2 Study population characteristics

\begin{tabular}{llll}
\hline Demographics & Consults & Alerts & Stats \\
\hline Age & Median 5.5 years $[<1,16]$ & \\
Gender & 143 males, 72 females & \\
ISS & $7.7[1,16]$ & $8.8[1,17]$ & $17[3,31]$ \\
Mechanisms of injury & & & \\
Fall & 37 & 11 & 9 \\
MVC & 9 & 43 & 19 \\
MVC-pedestrian & 1 & 27 & 7 \\
NAT & 9 & 2 & 12 \\
Sports injury & 5 & 0 & 3 \\
Struck with object & 8 & 6 & 6 \\
Animal bite & 4 & 3 & 1 \\
GSW & 2 & 0 & 3 \\
Bike accident & 0 & 9 & 1 \\
Other & 5 & 9 & 2 \\
\hline SS Inury Severity Score & $M$ mot & & \\
\hline
\end{tabular}

ISS Injury Severity Score, MVC motor vehicle collision, NAT non-accidental trauma, GSW gunshot wound 


\section{Main results}

On initial analysis, it was evident the NAT cohort as a group was exposed to more radiation than other trauma patients. To avoid inflating the average radiation doses for each activation status based on the number of NATs in each subcategory, we analyzed radiation exposure per patient in two subsets: (1) trauma patients excluding NATs and (2) NATs for all three activation statuses.

\section{Trauma patients excluding NATs}

Trauma stat patients received the most radiation followed by alerts when compared to trauma consults for CT dose and total number of CT scans $(p<0.001)$ (Table 3 ). The increase in radiation dose positively correlated with higher activation status and is best exemplified by the CT dose factor (Table 3). The CT dose factor represents the relative radiation due to $\mathrm{CT}$ scans as a factor of the CT dose for consults $(0.79 \mathrm{mSv})$. A 10 -fold increase between consults and stats was demonstrated by the CT dose factor.

\section{Non-accidental traumas}

Overall, NAT evaluations had more imaging. NAT consults and stats had more CT scans than their non-NAT counterparts. In fact, the average NAT consult was subject to four times as many CTs as the average trauma consult (Table $3, p<0.05$ ). The number of CTs generally increased with activation status (Fig. 1 and Table 3), although the number of CTs for NAT alerts (1.0) was similar to CTs per NAT consult (1.2). Total CT dose per patient (Table 3) increased with higher activation status. Specifically, NAT stats had a CT dose factor close to 12 .

\section{CT use across entire cohort \\ Repeated imaging}

Analyzing imaging patterns in the entire study population, the greatest variation in the number of CTs was noted among the stats; number of CTs ranged from 0 to 10 with $2-3$ CTs performed in $35 \%$ of the stat patients and 4-10 CTs in $40 \%$ of the stat patients per evaluation. The studies most often repeated across all activation statuses were head CTs, face/sinus CTs, and neck CTs with repetition rates of $45,13.8$, and $10 \%$ respectively.

\section{Dosing per scan}

Sixty-six percent $(40 / 61)$ of outside CT scans delivered more radiation than the pediatric protocol CT scans, of which $50.0 \%$ of the doses were at least double the standard pediatric dosage delivered at the children's hospital. A subset analysis of all patients who received OSH imaging $(N=30)$ was performed (Table 4$)$. The highest average OSH CT dose was noted in alerts $(9.77 \mathrm{mSV})$, followed by consults $(7.97 \mathrm{mSv})$, and with stats having the lowest dose (4.77 mSv). Analysis of different CT studies was not performed due to insufficient numbers for meaningful results in each separate category of CT scans.

\section{Plain films}

An increase in the number of plain films per patient with higher activation status was noted in both general trauma patients and NATs. NAT alerts (27.5 plain films) were typically similar to NAT consults (28 plain films) (Table 3). The majority of these plain films in NAT patients are accounted for by the routine ordering of a skeletal survey which typically consists of 23-24 plain films. Most plain films in addition to the first 23-24 films comprised repeat full body scans or were follow-up plain films of body regions where injuries were highly suspected or identified.

\section{Limitations}

There were several limitations in this study. Firstly, the mean effective radiation doses calculated were adjusted with conversion factors dependent on patient age and an

Table 3 Radiation exposure per trauma patient

\begin{tabular}{|c|c|c|c|c|c|c|c|}
\hline Activation status & Number & CT/patient* & $C T /$ patient $p$ value & $\mathrm{CT}$ dose $(\mathrm{mSv}) *+$ & $\mathrm{CT}$ dose factor & CT dose $p$ value & X-rays/patient \\
\hline \multicolumn{8}{|c|}{ Trauma patients excluding non-accidental traumas (NATs) } \\
\hline Consults & 61 & 0.3 & N/A & $0.79 \pm 2.2$ & 1 & N/A & 5.5 \\
\hline Alerts & 83 & 1.6 & $<0.001$ & $5.34 \pm 6.6$ & 6.76 & $<0.001$ & 8.4 \\
\hline Stats & 48 & 2.4 & $<0.001$ & $8.00 \pm 8.4$ & 10.13 & $<0.001$ & 13.7 \\
\hline \multicolumn{8}{|l|}{ NATs } \\
\hline Consults & 9 & 1.2 & 0.002 & $2.83 \pm 1.8$ & 3.58 & 0.009 & 28 \\
\hline Alerts & 2 & 1 & N/A & $3.28 \pm 1.5$ & 4.15 & N/A & 27.5 \\
\hline Stats & 12 & 3 & $<0.001$ & $9.19 \pm 5.0$ & 11.63 & $<0.001$ & 38.7 \\
\hline
\end{tabular}

$p$ values were computed for each group relative to trauma consult patients. $T$ tests were not performed comparing NAT alerts with trauma consults due to small sample size

*All values are averages

†All radiation doses include \pm SD and are calculated in milliSieverts (mSv) 
Number of CT scans per Trauma Patient

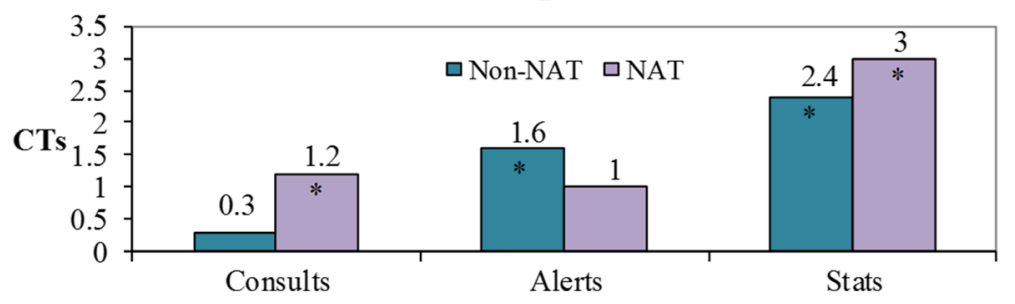

Fig. 1 Number of CT scans per trauma. *Groups that were found to have statistically significant increases in the number of $C T$ scans relative to the number of CTs performed in trauma consults. Note: A T test was not performed comparing NAT alerts with trauma consults due to small sample size

average body weight for that age. As a result, the doses provided may overestimate the doses in underweight patients and underestimate doses in overweight or obese patients. However, all current studies are held to this limitation. Additionally, different sets of conversion factors exist in the literature for determining mean effective dose with all resulting in slightly different dosage values [16] but are grossly similar. Alternative calculation methods may report values to be as much as $10.2 \pm$ $10.1 \%$ lower to $28 \pm 37.3 \%$ higher than the method used in this study [19]. Hence, the specific method used must be reported by each study in order to correctly compare the results across studies. Since our study was retrospective, another key limitation was the inability to definitely determine why some of the patients were re-scanned. Although it may be evident for some that clinical deterioration was the main cause based on medical record notes, it is difficult to differentiate between some causes such as poor scan quality of an OSH scan, redundant scanning given lack of accessibility of outside imaging, as opposed to lost/un-transferred disks in a retrospective analysis.

\section{Discussion}

Pediatric trauma patients encompass a population particularly prone to radiation exposure, given their increased susceptibility to ionizing radiation, and often receive care at non-pediatric hospitals which may not have attention to pediatric protocol dosing. Given the broad acceptance of non-operative management in trauma, there is a reasonable concern whether such widespread use of advanced imaging is warranted in all pediatric patients, particularly since surgical decisionmaking and interventions are now primarily driven by the physiologic status of the patients. Specifically, studies have shown that $\mathrm{CT}$ scans rarely influence the decision

Table 4 Outside hospital imaging

\begin{tabular}{llll}
\hline & Consults & Alerts & Stats \\
\hline No. of patients with OSH imaging & 10 & 14 & 16 \\
Average total OSH CT dose (mSv) & 7.97 & 9.77 & 4.77 \\
\hline
\end{tabular}

for operative intervention in trauma patients such as those who have sustained blunt abdominal trauma [20]. In patients who have been managed non-operatively, CT scans have shown limited value in directing further management and follow-up [21].

While an increase in imaging with a higher activation status appears intuitive, the CT dose factor reflecting the 10-fold CT dose increase between general trauma consults and general stats and a similar near 12-fold increase between general consults and NAT stats are both completely unexpected and grossly exceeded our expectations. A National Academy of Science report has estimated that children aged 15 and below have a $40 \%$ increase in cancer rate with exposures in the range of 10 to $20 \mathrm{mSv}$ [22]. Twenty-one percent of our trauma alerts and stats received doses within this range for a single trauma evaluation which is a very important baseline determination for future epidemiologic analysis. Furthermore, children with multiple trauma evaluations would be at even higher risk over time. The sharp increase in radiation exposure with activation status may be a result of (i) a higher index of suspicion in higher activation statuses, (ii) imaging decisions based on the presumption of injuries by mechanisms, and (iii) the pressure to identify all injuries expeditiously in the initial trauma evaluation.

Identifying the actual radiation doses are important to determine the actual exposures our population of children is receiving. Prior trauma studies have analyzed average radiation exposure based on estimations averaged across all levels of traumas extrapolated from the literature. The largest study queried the NTDB in 2010 included 84,863 patients and showed the mean effective $\mathrm{CT}$ radiation dose to be $12.0 \mathrm{mSv}^{15}$; however, the radiation doses were not based on actual radiologic records and were actually extrapolated from the literature. Also with such huge numbers of traumas that are not differentiated out by level of severity, it is easy to recognize that the simple trauma consults or low grade traumas would outnumber the more severe ones and thus power this finding to underestimate the amount of imaging a moderate or severe trauma work-up needs. Clearly with one trauma being $12 \mathrm{mSV}$, then, a child with just one 
trauma evaluation would potentially be in the concerning zone of cumulative radiation exposure. Our study shows that the real exposure is much higher for a stat activation so averaging all trauma activations may not give a clear accounting of which traumas actually receive a concerning amount of radiation. The National Survey of Children's Health (NSCH) has reported that $22.6 \%$ of children have over two serious trauma evaluations so the impact of higher radiation dosing on top of multiple trauma evaluations has serious epidemiologic consequences [23]. Additionally, the study [15] excluded patients who were not imaged within the first $24 \mathrm{~h}$ of admission and did not account for (i) those who were imaged subsequently and (ii) the less worrisome traumas who were not imaged at all, as opposed to our study which included the entirety of imaging for all patients during the full encounter including $\mathrm{OSH}$ imaging.

The subset analysis of NAT patients in our study provided a focus on a less studied patient group. A recent study has shown that 6.2 out of 100,000 children under the age of 18 have endured severe physical abuse [24]. Furthermore, this rate is nine times higher in patients under the age of one [24]. Since these patients suffer polytrauma, they are particularly prone to more frequent trauma evaluations and more extensive imaging compared to the corresponding cohort in the same activation status. The reason for extensive imaging appears bipartite. First, given the higher likelihood of multiple injuries, the pre-existing high level of suspicion in trauma patients is further justified. Second, positive imaging findings serve as evidentiary proof in investigations once legal action is pursued. While a recent study has stressed the importance of surgical evaluations in non-accidental traumas [25], a detailed analysis of radiation exposure in this subset has yet to be conducted.

Additionally, it is important to understand the significance of the skeletal surveys performed in NAT patients which utilizes 23-24 plain films. The amount of exposure is diminishingly small since each plain film of the abdomen/pelvis accounts for a mean effective dose of only $0.015 \mathrm{mSv}$ in a newborn to $0.05 \mathrm{mSv}$ in a 15 -yearold [26]. While X-ray scans impart significantly less radiation than CT scans, their high utilization demonstrates the comprehensive nature of imaging to prove a NAT case for legal intervention.

An advantage of our study was the inclusion of outside hospital (OSH) imaging which was uploaded to our imaging database. As a result, we were able to note the incidence of repeated imaging upon transfer and compare actual outside hospital radiation dosing with pediatric dosing at our pediatric center. Although overall and children's hospital CT doses correlated with activation status, we found that OSH CT doses did not have a similar correlation. Instead, trauma alerts received the most radiation followed by consults and then trauma stats. Alerts may have received the most radiation as $\mathrm{OSH}$ physicians may have viewed them as a group of patients just stable enough to be managed without specialist consultation at a children's hospital. As a result, they likely performed a complete work-up and later realized they needed to transfer the patients to a children's hospital. Presumably, the lowest CT dose in the trauma stats group is likely related to the realization that the patient should not be managed at the $\mathrm{OSH}$ and required transfer soon after the initial images were obtained.

The redundancy in imaging upon transfer was also shown in a prior study in which a high incidence of repeated CT scans in transferred trauma patients along with higher hospitalization costs were reported [27]. This is particularly concerning since the reason for reimaging in these patients reflects the lack of coordination between hospitals and timely access to transferred imaging studies. ED/trauma staff must advocate for the establishment of timely communication between major trauma centers and outside hospitals to reduce the delivery of excess radiation.

Our study had the additional advantage of evaluating the full hospital stay as opposed to initial evaluations limited to the first $24 \mathrm{~h}$. There is currently no data in the literature explicitly reflecting the propensity of repeated imaging of admitted trauma patients over the entire hospital stay. Our study showed that patients who received multiple $\mathrm{CT}$ scans as well as repeat full body plain film scans were often those who were hospitalized.

A prior study by Groner et al., [28] has suggested that traumas worked up by an emergency medicine attending physician are less likely to receive excessive radiation compared to an on-call trauma surgery resident who has less experience and is not constantly present with the patient. While this study highlighted only moderate level traumas at the authors' institution, such a model may benefit lower and higher activation statuses as well. As such, we advocate further education of surgical trainees and the new trauma model [28] that proposes that trauma surgeons should play the role of consultants rather than primary responders. Our study highlights the importance of adherence to the principle of maintaining radiation exposure "as low as reasonably achievable" (ALARA) [29]. The ALARA principles involve four cornerstones to decrease radiation dosimetry: (1) use weight-based protocols, (2) consider alternative nonradiating modalities, (3) use focused or limited-view studies when clinically appropriate, and (4) dissuade repeat $\mathrm{CT}$ studies.

These strategies advocate low-dose pediatric-specific protocols and other techniques such as flash CT, minimizing thin-cut $\mathrm{CT}$ imaging, utilizing "justification and optimization" determinations in granting CT requests 
(to avoid additional or unnecessary radiation exposure), and dedicated pediatric CT imaging services with pediatric-specific CT technologists to improve compliance with adjusted lower CT exposure parameters and lower estimated effective doses of radiation delivered to pediatric patients. These ALARA strategies have resulted in much greater compliance with pediatric dose-adjusted $\mathrm{CT}$ protocols and well-recognized reduced radiation exposure to patients.

The majority of pediatric trauma patients are managed at non-pediatric (adult) trauma hospitals, since children are quickly transported to the nearest hospital initially for evaluation and thus are exposed to twice the radiation dose that they would have been exposed to in a pediatric hospital with a pediatric CT imaging dose reduction protocol in effect [30]. Moreover, in an age during which the paradigm of "pan-scanning" is common practice, we must enforce protocols to minimize radiation exposure in our patients. For this to occur, the entire multidisciplinary team including ED staff, pediatric/trauma surgeons, and radiologists must be on board.

\section{Conclusions}

In summary, this study is the first to correlate the degree of radiation exposure with trauma activation status defining actual average radiation exposure values for each subset. The identified factors associated with the most radiation include stat activations, suspected NATs, and trauma patients transferred after OSH imaging due to tendency to use adult dosing parameters on pediatric trauma patrons. To minimize the radiation footprint, we may need to change the current practice of widespread utilization of advanced imaging to identify all possible injuries regardless of symptoms.

\section{Acknowledgements}

We thank Jeannie Joglar in the Department of Radiology at Children's Medical Center for providing radiologic background for this study.

\section{Authors' contributions \\ ZF, ZF, and ACF conceived and designed the study and ACF supervised the data collection and management on-site. RMS provided statistical advice on study design and analyses and ACF, ZF and ZF, worked with the Trauma Registry administrators to conduct the de-identified analyses. RMS, ZF, ZF, and ACF drafted the article, and all the authors contributed substantially to its revision. All authors provided feedback on the study design, had full access to all the data in the study, and had final responsibility for the decision to submit for publication. ACF and RMS take responsibility for the paper as a whole. All authors read and approved the final manuscript.}

\section{Competing interest}

The authors declare that they have no competing interests.

\section{Publisher's Note}

Springer Nature remains neutral with regard to jurisdictional claims in published maps and institutional affiliations.

\section{Author details}

${ }^{1}$ The Heller School for Social Policy and Management, Brandeis University, Waltham, MA, USA. ${ }^{2}$ Department of Otolaryngology, University North
Carolina, Children's Hospital, 101 Manning Drive, Chapel Hill, NC, USA ${ }^{3}$ Department of Neurology, MedStar Georgetown University Hospital, Reservoir Rd NW, Washington, DC 3800, USA. ${ }^{4}$ Florida Atlantic University/St. Mary's Medical Center, 927 45th Street, Suite 301, West Palm Beach, FL 33407, USA.

Received: 20 December 2017 Accepted: 15 February 2018

Published online: 14 March 2018

\section{References}

1. Brenner DJ, Hall EJ. Computed tomography—an increasing source of radiation exposure. N Engl J Med. 2007;357:2277-84.

2. International Marketing Ventures (IMV) 2014 CT market summary report. IMV Medical Information Division. Des Plains, Illinois, 2014.

3. Ron E. lonizing radiation and cancer risk: evidence from epidemiology. Pediatr Radiol. 2002;32:232-7.

4. Berrington de González A, Mahesh M, Kim KP, et al. Projected cancer risks from computed tomographic scans performed in the United States in 2007. Arch Intern Med. 2009;169:2071-7.

5. Brody AS, Frush DP, Huda W, Brent RL. Radiation risk to children from computed tomography. Pediatrics. 2007;120:677-82.

6. Brenner D, Elliston C, Hall E, Berdon W. Estimated risks of radiation-induced fatal cancer from pediatric CT. AJR Am J Roentgenol. 2001;176:289-96.

7. Hall EJ, Brenner DJ. Cancer risks from diagnostic radiology. Br J Radiol. 2008; 81(965):362-78.

8. Berdon WE, Slovis TL. Where we are since ALARA and the series of articles on $\mathrm{CT}$ dose in children and risk of long-term cancers: what has changed? Pediatr Radiol. 2002;32(10):699.

9. Pearce MS, Salotti JA, Little MP, et al. Radiation exposure from CT scans in childhood and subsequent risk of leukaemia and brain tumours: a retrospective cohort study. Lancet. 2012;380:499-505.

10. Carr BG, Nance ML. Access to pediatric trauma care: alignment of providers and health systems. Curr Opin Pediatr. 2010;22(3):326-31.

11. WISQARS (Web-based Injury Statistics Query and Reporting System) -CDC. https://www.cdc.gov/injury/wisqars/index.html. Accessed 5 Feb 2018.

12. American College of Surgeons Committee on Trauma. Advanced Trauma Life Support for Doctors (ATLS) student course manual. 8th ed. Chicago: American College of Surgeons; 2008. p. 225-45.

13. Inaba K, Branco BC, Lim G, et al. The increasing burden of radiation exposure in the management of trauma patients. J Trauma. 2011;70:1366-70.

14. Kim PK, Zhu X, Houseknecht E, Nickolaus D, Mahboubi S, Nance ML. Effective radiation dose from radiologic studies in pediatric trauma patients. World J Surg. 2005;29:1557-62.

15. Kharbanda AB, Flood A, Blumberg K, Kreykes NS. Analysis of radiation exposure among pediatric trauma patients at national trauma centers. J Trauma Acute Care Surg. 2013;74(3):907-11.

16. Brunetti MA, Mahesh M, Nabaweesi R, Locke P, Ziegfeld S, Brown R. Diagnostic radiation exposure in pediatric trauma patients. J Trauma. 2011;70(2):E24-8.

17. NCRP. lonizing radiation exposure of the population of the United States, NCRP Report No. 93. Bethesda: National Council on Radiation Protection and Measurements; 1987.

18. Shrimpton PC, Hillier MC, Lewis MA, Dunn M. National survey of doses from CT in the UK: 2003. Br J Radiol. 2006;79:968-80.

19. Newman B, Ganguly A, Kim JE, Robinson T. Comparison of different methods of calculating $C T$ radiation effective dose in children. AJR Am J Roentgenol. 2012;199(2):W232-9.

20. Ruess L, Sivit CJ, Eichelberger MR, Gotschall CS, Taylor GA. Blunt abdominal trauma in children: impact of $\mathrm{CT}$ on operative and nonoperative management. AJR Am J Roentgenol. 1997:169:1011-4.

21. Navarro O, Babyn PS, Pearl RH. The value of routine follow-up imaging in pediatric blunt liver trauma. Pediatr Radiol. 2000;30:546-50.

22. National Research Council. Health risks from exposure to low levels of ionizing radiation: BIER VII Phase 2. Washington, DC: National Academy Press; 2006

23. National Health Interview Survey. Maternal and Child Health Bureau in collaboration with the National Center for Health Statistics. [Insert SPSS/SAS] 2014 NHIS Child Data Set prepared by the Data Resource Center for Child and Adolescent Health, Child and Adolescent Health Measurement Initiative. 2014. http://www.childhealthdata.org/help/dataset/drc-ftp-2014nhis. Accessed 11 Mar 2018.

24. Leventhal JM, Martin KD, Gaither JR. Using US data to estimate the incidence of serious physical abuse in children. Pediatrics. 2012;129(3):458-64. 
25. Larimer EL, Fallon SC, Westfall J, Frost M, Wesson DE, Naik-Mathuria BJ. The importance of surgeon involvement in the evaluation of non-accidental trauma patients. J Pediatr Surg. 2013;48(6):1357-62.

26. Ahmed BA, Connolly BL, Shroff P, Chong AL, Gordon C, Grant R, et al. Cumulative effective doses from radiologic procedures for pediatric oncologic patients. Pediatrics. 2010;126(4):e851-8.

27. Jones AC, Woldemikael D, Fisher T, Hobbs GR, Prud'homme BJ, Bal GK. Repeated computed tomographic scans in transferred trauma patients: indications, costs, and radiation exposure. J Trauma Acute Care Surg. 2012;73:1564.

28. Groner Jl, Covert J, Lowell WL, et al. The impact of managing moderately injured pediatric trauma patients without immediate surgeon presence. J Pediatr Surg. 2007;42:1026-9.

29. Slovis TL, editor. ALARA conference proceedings. The ALARA concept in pediatric CT: intelligent dose reduction. Pediatr Radiol. 2002;32:217.

30. Nabaweesi $R$, et al. Injured children receive twice the radiation dose at nonpediatric trauma centers compared with pediatric trauma centers. J Am Coll Radiol. 2018;15:58-64. 